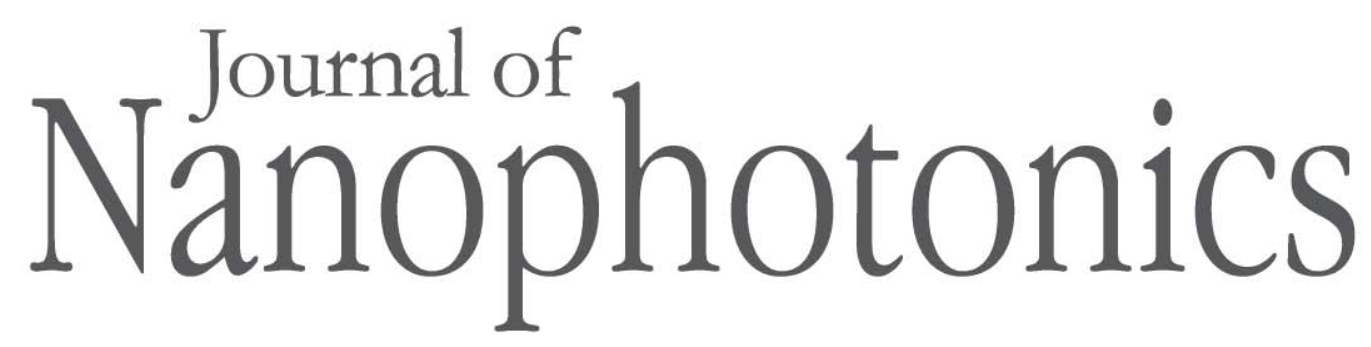

SPIEDigitalLibrary.org/jnp

\title{
Electric and magnetic dipolar response of germanium nanospheres: interference effects, scattering anisotropy, and optical forces
}

Raquel Gómez-Medina

Braulio García-Cámara

Irene Suárez-Lacalle

Francisco González

Fernando Moreno

Manuel Nieto-Vesperinas

Juan José Sáenz 


\title{
Electric and magnetic dipolar response of germanium nanospheres: interference effects, scattering anisotropy, and optical forces
}

\author{
Raquel Gómez-Medina, ${ }^{\mathrm{a}, \mathrm{b}}$ Braulio García-Cámara, ${ }^{\mathrm{c}}$ \\ Irene Suárez-Lacalle, ${ }^{a}$ Francisco González, ${ }^{c}$ Fernando Moreno, ${ }^{c}$ \\ Manuel Nieto-Vesperinas, ${ }^{b}$ and Juan José Sáenz ${ }^{a}$ \\ ${ }^{a}$ Universidad Autónoma de Madrid, Departamento de Física de la Materia Condensada, \\ 28049 Madrid, Spain \\ juanjo.saenz@uam.es \\ ${ }^{\mathrm{b}}$ Instituto de Ciencia de Materiales de Madrid, C.S.I.C., Campus de Cantoblanco, \\ 28049 Madrid, Spain \\ ${ }^{\mathrm{c}}$ Universidad de Cantabria, Departamento de Física Aplicada, Avda. de los Castros s/n, \\ 39005 Santander, Spain
}

\begin{abstract}
The coherent combination of electric and magnetic responses is the basis of the electromagnetic behavior of new engineered metamaterials. The basic constituents of their meta-atoms usually have metallic character and consequently high absorption losses. Based on standard "Mie" scattering theory, we found that there is a wide window in the near-infrared (wavelengths 1 to $3 \mu \mathrm{m}$ ), where light scattering by lossless submicrometer Ge spherical particles is fully described by their induced electric and magnetic dipoles. The interference between electric and magnetic dipolar fields is shown to lead to anisotropic angular distributions of scattered intensity, including zero backward and almost zero forward scattered intensities at specific wavelengths, which until recently was theoretically established only for hypothetically postulated magnetodielectric spheres. Although the scattering cross section at zero backward or forward scattering is exactly the same, radiation pressure forces are a factor of 3 higher in the zero forward condition. (c) 2011 Society of Photo-Optical Instrumentation Engineers (SPIE). [DOI: 10.1117/1.3603941]
\end{abstract}

Keywords: scattering; particles; anisotropic optical materials; magneto-optical materials; nanomaterials; resonators.

Paper 11059R received Apr. 17, 2011; revised manuscript received May 21, 2011; accepted for publication Jun. 3, 2011; published online Jun. 29, 2011.

\section{Introduction}

Electromagnetic scattering from nanometer-scale objects has long been a topic of large interest and relevance to fields from astrophysics or meteorology to biophysics, medicine, and material science. ${ }^{1-4}$ In the last few years, small particles with resonant magnetic properties are being explored as constitutive elements of new metamaterials and devices. Magnetic effects, however, cannot be easily exploited in the visible or infrared regions due to intrinsic natural limitations of optical materials and the quest for magnetic plasmons and magnetic resonant structures at optical frequencies ${ }^{5-7}$ has then been mainly focused on metallic structures. The unavoidable problems of losses and saturation effects inherent to these metamaterials in the optical and near-infrared regimes have stimulated the study of high-permittivity particles as their constitutive elements: ${ }^{8-18}$ For very large permittivities, small spherical particles present well defined sharp resonances; ${ }^{1}$ either electric or magnetic resonant responses can then be tuned by choosing the appropriate sphere radius. However, the applicability of conventional

$1934-2608 / 2011 / \$ 25.00$ @ 2011 SPIE 
homogenization procedures to determine the constitutive parameters of composites of resonant spheres cannot be implemented without due regard to their limitations on volume fraction and size parameter. ${ }^{19}$

In the presence of both electric and magnetic properties, the scattering characteristics of a small object present marked differences with respect to pure electric or magnetic responses. Even in the simplest case of small or dipolar scatterers, remarkable scattering effects of magnetodielectric particles were theoretically established by Kerker et al. ${ }^{20}$ concerning suppression or minimization of either forward or backward scattering. Intriguing applications in scattering cancellation and cloaking ${ }^{21-23}$ and magneto-optical systems, ${ }^{24-27}$ together with the unusual properties of the optical forces on magnetodielectric particles, ${ }^{28,29}$ have renewed interest in the field.

The striking characteristics of the scattering diagram of small (Rayleigh) magnetodielectric particles ${ }^{20,30,31}$ were obtained assuming arbitrary values of relative permittivity and permeability.

Nevertheless, no concrete example of such particles that might present those interesting properties in the visible or infrared regions had been proposed. Very recently, it has been shown ${ }^{32,33}$ that submicrometer silicon spheres present dipolar magnetic and electric responses, characterized by their respective first-order Mie coefficient, in the near-infrared, in such a way that either of them can be selected by choosing the illumination wavelength. In a later work, it has also been shown ${ }^{29,34}$ that $\mathrm{Si}$ spheres constitute such a previously quested real example of a dipolar particle with either electric and/or magnetic response, of consequences both for their emitted intensity and behavior under electromagnetic forces.

These properties should not be restricted to Si particles, but should also apply to other dielectric materials with relatively moderate refraction index. In the present work, we discuss the effects associated to the interference between electric and magnetic dipoles in germanium spheres. The paper is structured as follows. Based on the exact Mie theory, in Sec. 2 we show that both the extinction cross section and the scattering diagrams of submicrometer Ge spheres in the infrared region can be well described by dipolar electric and magnetic fields, being quadrupolar and higher order contributions negligible in this frequency range. Specifically, the scattering diagrams calculated at the generalized Kerker's (GK) conditions are shown to be equivalent to those previously reported ${ }^{20,31}$ for hypothetical magnetodielectric particles. Following previous work regarding the peculiar properties of optical forces at GK conditions, ${ }^{29}$ in Sec. 3 we analyze the consequences of the strong scattering anisotropy on the radiation pressure on Ge particles.

\section{Extinction Cross Sections and Scattering Anisotropy of Submicrometer Germanium Spheres}

Consider a nonabsorbing dielectric sphere of radius $a$ and permittivity $\epsilon_{p}=m_{p}^{2}$ in an otherwise uniform medium with real relative permittivity $\epsilon$ and refractive index $m=\sqrt{\epsilon \mu}$. The relative permeability $\mu_{p}$ of the sphere and of the surrounding medium, $\mu$, are both assumed to be $\mu_{p}=\mu=1$ (even though in some of the equations to be written in this paper, $\mu$ will explicitly be included for the sake of comprehensiveness of the theory).

Under plane wave illumination, and assuming linearly polarized light, the incident wave is described by

$$
E=E_{0} \mathbf{u}_{x} e^{i k z} e^{-i \omega t}, \quad B=B_{0} \mathbf{u}_{y} e^{i k x} e^{-i \omega t}
$$

where $k=m \omega / c=m 2 \pi / \lambda, \lambda$ being the wavelength in vacuum and $B_{0}=m E_{0}$. The field scattered by the sphere can be decomposed into a multipole series (that which is called Mie's expansion) characterized by the $\left\{a_{n}\right\}$ electric and $\left\{b_{n}\right\}$ magnetic Mie coefficients (being $a_{1}$ and $b_{1}$ proportional to the electric and magnetic dipoles, $a_{2}$ and $b_{2}$ to the quadrupoles, and so on). 
Gómez-Medina et al.: Electric and magnetic dipolar response of germanium nanospheres: interference effects...

Mie coefficients are related to the scattering phase-shifts $\xi_{n}$ and $\beta_{n}$ through $^{1}$

$$
\begin{aligned}
& a_{n}=\frac{1}{2}\left(1-e^{-2 i \xi_{n}}\right)=i \sin \xi_{n} e^{-i \xi_{n}}, \\
& b_{n}=\frac{1}{2}\left(1-e^{-2 i \beta_{n}}\right)=i \sin \beta_{n} e^{-i \beta_{n}} .
\end{aligned}
$$

The extinction, $Q_{\text {ext }}$, and scattering, $Q_{S}$, cross sections of a dielectric sphere, expressed in the Mie coefficients, read

$$
\begin{aligned}
& Q_{\mathrm{ext}}=\frac{2 \pi}{k^{2}} \sum_{n=1}^{\infty}(2 n+1) \operatorname{Re}\left\{a_{n}+b_{n}\right\}, \\
& Q_{S}=\frac{2 \pi}{k^{2}} \sum_{n=1}^{\infty}(2 n+1)\left\{\left|a_{n}\right|^{2}+\left|b_{n}\right|^{2}\right\} .
\end{aligned}
$$

In absence of absorption, i.e., for real $m, m_{p}, Q_{\text {ext }}=Q_{s}$.

In the small particle limit $(x \equiv k a \ll 1)$ and large particle permittivities $\left(m_{p} / m \gg 1\right)$ the extinction cross section presents characteristic sharp resonance peaks. At each resonance, the extinction cross section is of the order of $\lambda^{2}$ and it is independent of the particle size or refractive index. ${ }^{1}$ Interesting applications of well defined Mie resonances, associated to low loss and large dielectric constants, are accessible for different materials at microwave and terahertz frequencies. However, as $m_{p}$ decreases there is an increasing overlap between the cross section peaks, and the resonant character weakens until it disappears. Since usually nonabsorbing materials present low refractive index in the near-infrared and visible frequency ranges, Mie resonances of small particles in these regimes have not been considered in detail. However, a recent analysis of the cross section of submicrometer dielectric particles ${ }^{33}$ show that well defined resonances can be found for materials with relative refractive index as low as $m_{p} \sim 3 m$.

Germanium submicrometer particles are a good candidate to explore the effects associated to the interference between electric and magnetic responses. In the micrometer wavelength regime, within the transparent region of germanium $(\lambda \gtrsim 1.4 \mu \mathrm{m})$, the refraction index can be well approximated by a real constant $m_{p} \approx \sqrt{16}=4$ (see, for example, Ref. 35). The calculated exact extinction (or scattering) cross section of a $240 \mathrm{~nm}$ radius Ge sphere in vacuum $(m=1)$ is plotted in Fig. 1. Although there is an overlap between the first dipolar peaks, the first dipolar magnetic resonance (at $\lambda \approx 2000 \mathrm{~nm}$ ) and the electric dipolar resonance (at $\lambda \approx 1500 \mathrm{~nm}$ ) are

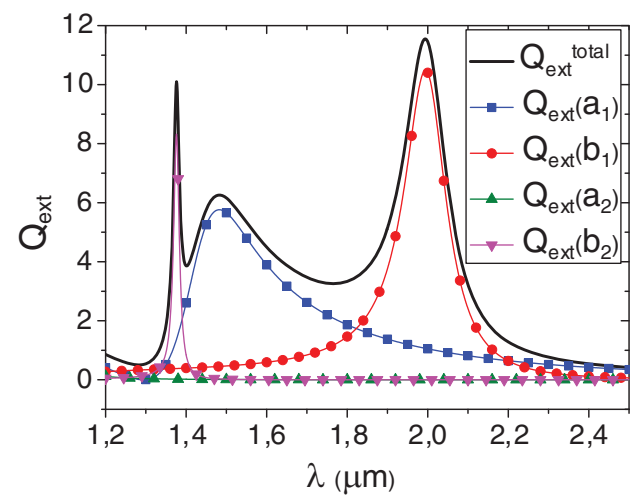

Fig. 1 Extinction cross section $Q_{\mathrm{ext}}$ versus the wavelength $\lambda$ for a $240 \mathrm{~nm}$ Ge sphere (the refraction index $m_{p}=4$ is constant and real in this wavelength range). The contribution of each term in the Mie expansion is also shown. The red line corresponds to the magnetic dipole contribution. 
still very well defined. As we will see, this overlap plays a key role in determining the peculiar scattering diagrams of Ge particles. Interestingly, for wavelengths larger than $\lambda \approx 1400 \mathrm{~nm}$, the cross section is completely determined by the first $b_{1}$ and $a_{1}$ coefficients. Therefore, Eq. (4) becomes

$$
Q_{\mathrm{ext}}=Q_{S} \approx \frac{6 \pi}{k^{2}} \operatorname{Re}\left\{a_{1}+b_{1}\right\}
$$

where $a_{1}$ and $b_{1}$ are the exact (nonapproximated) dipolar electric and magnetic Mie coefficients, respectively. ${ }^{1}$ In other words, in this regime submicrometer Ge particles can be treated as dipolar particles in the sense that only the dipolar term of Eq. (4) contributes to the scattered field.

Dipolar particles are usually characterized by their electric and/or magnetic complex polarizabilities, $\alpha_{e}$ and $\alpha_{m}$, which may be written in the form ${ }^{28,33}$

where

$$
\begin{gathered}
\alpha_{e}=3 i \epsilon a_{1} /\left(2 k^{3}\right)=\frac{\alpha_{e}^{(0)}}{1-i \frac{2}{3 \epsilon} k^{3} \alpha_{e}^{(0)}}, \\
\alpha_{m}=3 i b_{1} /\left(2 \mu k^{3}\right)=\frac{\alpha_{m}^{(0)}}{1-i \frac{2}{3} \mu k^{3} \alpha_{m}^{(0)}} .
\end{gathered}
$$

$$
\alpha_{e}^{(0)}=-\frac{3 \epsilon}{2 k^{3}} \tan \xi_{1}, \quad \alpha_{m}^{(0)}=-\frac{3}{2 \mu k^{3}} \tan \beta_{1} .
$$

In the absence of absorption, $\alpha_{e}^{(0)}$ and $\alpha_{m}^{(0)}$ are real quantities.

For a pure electric or a pure magnetic dipole, in the absence of interferences, the far field radiation pattern is symmetrically distributed between forward and backward scattering. However, when we consider the coherent contribution of both electric and magnetic dipoles, the radiation pattern is mainly distributed in the forward or backward region according to whether $\Re\left(\alpha_{e} \alpha_{m}^{*}\right)$ is positive or negative, respectively. ${ }^{28,29}$ Interestingly, at the GK conditions $\left|\epsilon^{-1} \alpha_{e}\right|^{2}=\left|\mu \alpha_{m}\right|^{2}$, the scattered intensity should be independent of the incident polarization angle:

$$
\frac{d Q_{S}}{d \Omega}(\theta)=k^{4} \mid \epsilon^{-1} \alpha_{e} \|^{2}\left(1+\cos ^{2} \theta\right)+2 k^{4} \frac{\mu}{\epsilon} \Re\left(\alpha_{e} \alpha_{m}^{*}\right) \cos \theta .
$$

The interference between electric and magnetic dipoles lead to a number of interesting effects:

i) The intensity in the backscattering direction can be exactly zero whenever $a_{1}=b_{1}$ or

$$
\epsilon^{-1} \alpha_{e}=\mu \alpha_{m} ; \quad \frac{d Q_{s}}{d \Omega}\left(180^{\circ}\right)=0 .
$$

This anomaly was theoretically predicted for magnetodielectric particles and takes place at the first Kerker condition: ${ }^{20} \epsilon_{p} / \epsilon=\mu_{p} / \mu$. Equation (11) can be considered as a generalized first Kerker condition for arbitrary dipolar particles.

ii) Although the intensity cannot be exactly zero in the forward direction (causality imposes $\Im\left\{\alpha_{e}\right\}, \Im\left\{\alpha_{m}\right\}>0$ ), in absence of particle absorption, the forward intensity presents a minimum at ${ }^{29}$

$$
\begin{gathered}
\Re\left\{\epsilon^{-1} \alpha_{e}\right\}=-\Re\left\{\mu \alpha_{m}\right\} \quad \text { and } \Im\left\{\epsilon^{-1} \alpha_{e}\right\}=\Im\left\{\mu \alpha_{m}\right\} \\
\frac{d Q_{S}}{d \Omega}\left(0^{\circ}\right)=\frac{16}{9} k^{10}\left|\epsilon^{-1} \alpha_{e}\right|^{4} .
\end{gathered}
$$

The first line of Eq. (12) is considered as a generalized second Kerker condition. In the particular case of lossless magnetodielectric particles in the small-particle limit (i.e., when the 


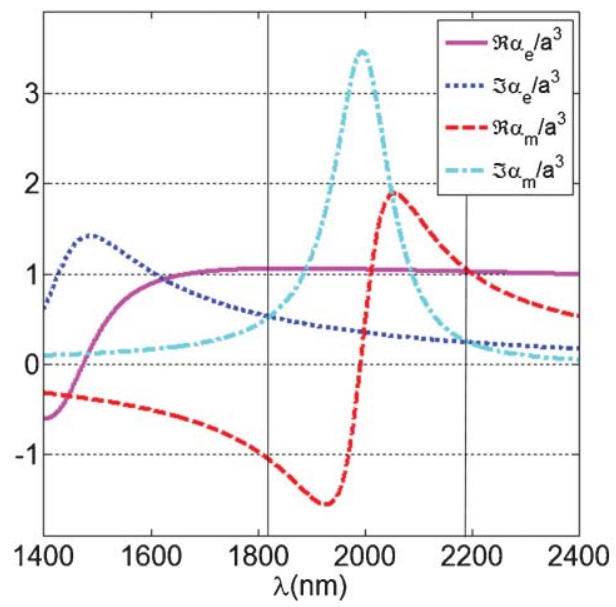

Fig. 2 Normalized real and imaginary parts of both the electric and magnetic polarizabilities for a Ge sphere of radius $a=240 \mathrm{~nm} ; \epsilon_{p}=16$ and $\mu_{p}=1$. The host medium has $\epsilon=\mu=1$. The first and second GK conditions (at $\lambda=2193$ and $\lambda=1823 \mathrm{~nm}$, respectively) are marked by the right and left vertical lines, respectively.

coefficients $a_{1}$ and $b_{1}$ can be approximated by the first terms in the expansions of these coefficients in powers of the size parameter, $x$ ) this happens at the second Kerker condition: ${ }^{20,31} \epsilon_{p} / \epsilon$ $=-\left(\mu_{p} / \mu-4\right) /\left(2 \mu_{p} / \mu+1\right)$. Although the original derivation ${ }^{20}$ was obtained in the quasistatic approximation $\left(\Im\{\alpha\} \approx 0\right.$, thus leading to $d Q\left(0^{\circ}\right) / d \Omega=0$ ), the actual intensity for a very small particle goes as $\sim(k a)^{10}$ which, for Rayleigh particles, would be negligible. ${ }^{23}$ However, the derivation of the GK conditions (11) and (12) with unusual scattering properties was obtained $^{29}$ with the unique assumption that the radiation fields are well described by dipolar electric and magnetic fields (i.e., using the exact expressions for $a_{1}$ and $b_{1}$ which adequately describe the scattered field). This goes well beyond the Rayleigh limit and should apply to any dipolar particle. Specifically, the GK conditions also apply to purely dielectric spheres $\left(\mu_{p}=1\right)$ providing that their scattering properties may be described by the two first terms in the Mie expansion.

Figure 2 shows the real and imaginary parts of the polarizabilities [Eq. (8)] for a Ge sphere with $a=240 \mathrm{~nm}$. One sees the values of $\lambda$ at which $\Im\left\{\alpha_{e}\right\}=\Im\left\{\alpha_{m}\right\}$, which are where the first and second GK conditions hold for these polarizabilities.

In order to check the validity of the dipolar approach and confirm the predictions at the GK conditions, we have computed the exact scattering diagram from the full Mie expansion. The numerical results are shown in Fig. 3. While the backward intensity drops to zero at the first GK condition wavelength, at the second condition, although the most of the intensity goes backward, the scattering diagram presents a very small peak in the forward direction. As expected from the extinction cross section, the far field pattern is fully consistent with the dipolar approximation. In particular, at the GK conditions it is independent of the incoming polarization. Dielectric spheres and, in particular, lossless Ge particles in the near-infrared, then constitute a realizable laboratory to observe such interesting scattering effects.

\section{Effects on Optical Forces}

Let us now discuss some of the consequences of the GK condition on optical forces. The theory of the force on a dipolar magnetic particle has recently been developed. ${ }^{28,36}$ This includes pure dielectric particles which can be well described by its first two electric and magnetic Mie coefficients. ${ }^{28}$ The time averaged force on a dipolar particle can be written as the sum 

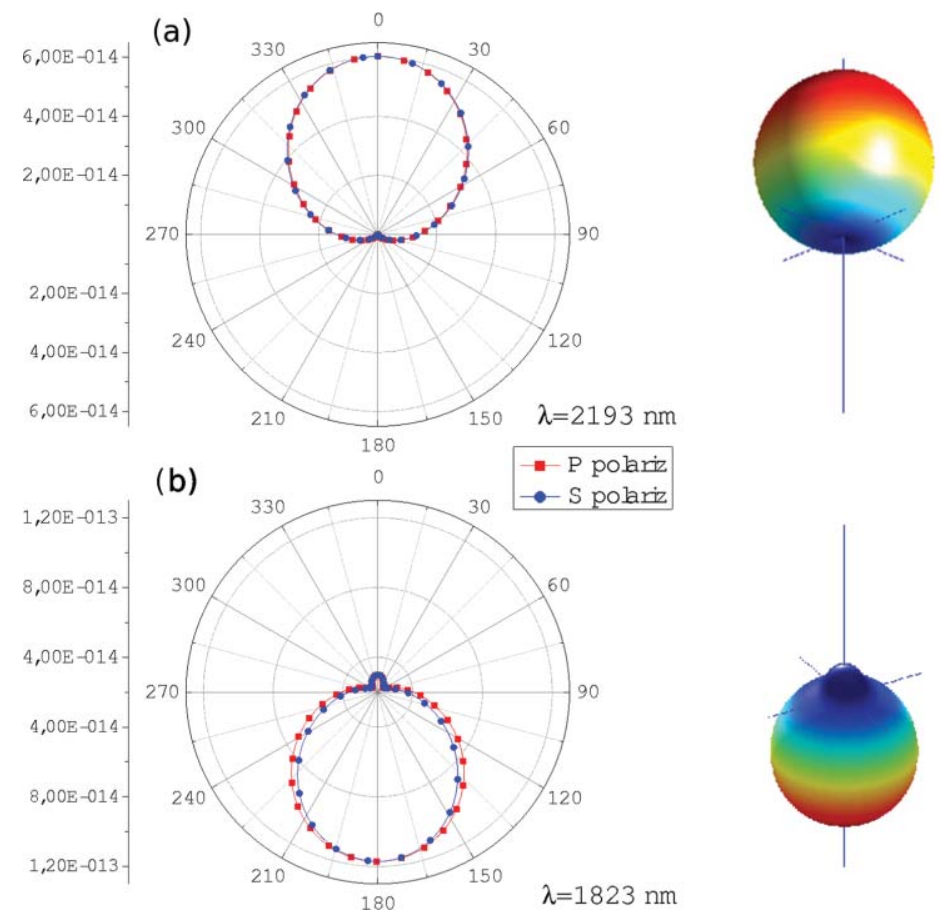

Fig. 3 Scattering diagrams for the $240 \mathrm{~nm}$ Ge nanoparticle of Fig. 1. Both polarizations, with the incident electric field parallel (TM or P polarization) or normal (TE or S polarization) to the scattering plane are considered.

of three terms: ${ }^{28}$

$$
\begin{aligned}
\langle\mathbf{F}\rangle & =\left\langle\mathbf{F}_{e}\right\rangle+\left\langle\mathbf{F}_{m}\right\rangle+\left\langle\mathbf{F}_{e-m}\right\rangle \\
& =\mathbf{u}_{z} F_{0}\left[\frac{1}{a^{3}} \Im\left\{\epsilon^{-1} \alpha_{e}+\mu \alpha_{m}\right\}-\frac{2 k^{3}}{3 a^{3}} \frac{\mu}{\epsilon} \Re\left(\alpha_{e} \alpha_{m}^{*}\right)\right],
\end{aligned}
$$

where $F_{0} \equiv \epsilon k a^{3}\left|E_{0}\right|^{2} / 2$. The first two terms, $\left\langle\mathbf{F}_{e}\right\rangle$ and $\left\langle\mathbf{F}_{m}\right\rangle$, correspond to the forces on the induced pure electric and magnetic dipoles, respectively. $\left\langle\mathbf{F}_{e-m}\right\rangle$, due to the interaction between both dipoles, ${ }^{28,36}$ is related to the asymmetry in the scattered intensity distribution [cf. the last term in Eq. (10)]. ${ }^{28}$

At the first GK condition, Eq. (11), the interference term of Eq. (13) cancels out the magnetic contribution and we obtain $\langle\mathbf{F}\rangle=\left\langle\mathbf{F}_{e}\right\rangle$. At the second GK condition, Eq. (12), where the backscattering is enhanced, $\langle\mathbf{F}\rangle=3\left\langle\mathbf{F}_{e}\right\rangle$. Notice that at both GK conditions the scattering cross section is exactly the same; however, the radiation pressures differ by a factor of 3 . These properties are illustrated in Fig. 4, where we show the different contributions to the total time averaged force on a submicrometer Ge particle.

The strong peak in the radiation pressure force is mainly dominated by the first "magnetic" Mie resonance. This is striking and in contrast with all previous beliefs about optical forces on dipolar dielectric particles, that assumed that these forces would solely be described by the electric polarizability. It is also common to assume that for dielectric particles the real part of the polarizability is much larger than its imaginary part. As a matter of fact, this is behind the development of optical tweezers, in which gradient forces (that are proportional to $\Re\left\{\alpha_{e}\right\}$ ), dominate over the radiation pressure or scattering force contribution (which is proportional to $\left.\Im\left\{\alpha_{e}\right\}\right) .{ }^{37}$ However, as the size of the particle increases, and for any dielectric particle, there is a crossover from electric to magnetic response as we approach the first Mie 


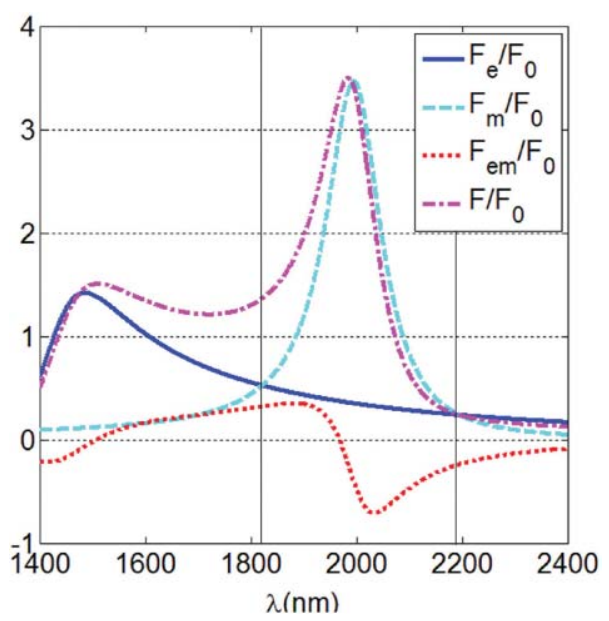

Fig. 4 Different contributions to the total radiation pressure versus the wavelength, for the $\mathrm{Ge}$ particle of Fig. 1. Normalization is done by $F_{0}=k \epsilon a^{3}\left|E_{0}\right|^{2} / 2$. Again, the vertical lines mark the first and second GK conditions. Notice that when the first GK condition is fullfilled, i.e., $\Im \alpha_{e}=\Im \alpha_{m}$ and $\Re \alpha_{e}=\Re \alpha_{m},\langle F\rangle=\left\langle F_{e}\right\rangle=\left\langle F_{m}\right\rangle=-\left\langle F_{e-m}\right\rangle$.

resonance, the point at which the response is absolutely dominated by the magnetic dipole. Moreover, just at the resonance, and in absence of absorption, $\Re\left\{\alpha_{m}\right\}=0$ and $\Im\left\{\alpha_{m}\right\}$ $=3 /\left(\mu 2 k^{3}\right)$. Then, the radiation pressure contribution of the magnetic term dominates the total force $\langle\mathbf{F}\rangle \simeq\left\langle\mathbf{F}_{m}\right\rangle \approx\left(\epsilon\left|E_{0}\right|^{2} / 2\right)\left[3 /\left(2 k^{2}\right)\right]$. Namely, in resonance the radiation pressure force presents a strong peak, the maximum force being independent of both material parameters and particle radius.

\section{Conclusions}

In summary, we have predicted that real small dielectric particles made of nonmagnetic materials present interesting scattering properties similar to those previously reported for somewhat hypothetical magnetodielectric particles ${ }^{20}$ resulting from an interplay between real and imaginary parts of both electric and magnetic polarizabilities. The exact scattering diagram, computed from the full Mie expansion, of submicrometer Ge particles in the infrared, was shown to be consistent with the expected result for dipolar electric and magnetic scattering. Then we showed that these unusual scattering effects also affect the radiation pressure on these small particles; specifically, the generalized Kerker's conditions have been tested on Ge spheres. Submicrometer Ge particles constitute an excellent laboratory to observe such new scattering phenomena and force effects. Being Ge permittivitys is higher than $\mathrm{Si}$, the present work extends the range of some possible applications (previously suggested for silicon particles), to systems where the host medium presents refractive index larger than vacuum.

The extraordinary scattering properties discussed here will strongly affect the dynamics of particle confinement in optical traps and vortex lattices ${ }^{38}$ governed by both gradient and curl forces. ${ }^{28,39}$ The interference between electric and magnetic dipoles suggest also intriguing possibilities regarding resonant coupling between the scattered dipolar field and guided modes in confined geometries. ${ }^{40}$ We do believe that our results will stimulate further experimental and theoretical work in different directions, from optical trapping and particle manipulation to cloaking and the design of optical metamaterials based on lossless dielectric particles.

\section{Acknowledgments}

We appreciate interesting discussions with P. Albella, L. S. Froufe-Pérez, A. García-Etxarri, and J. M. Saiz. This work has been supported by the EU NMP3-SL-2008-214107-Nanomagma, 
Gómez-Medina et al.: Electric and magnetic dipolar response of germanium nanospheres: interference effects...

the Spanish MICINN Consolider NanoLight (CSD2007-00046), FIS2010-21984, FIS200913430-C01-C02, and FIS2007-60158, as well as by the Comunidad de Madrid MicroseresCM (S2009/TIC-1476). Work by R.G.-M. was supported by the MICINN "Juan de la Cierva" Fellowship.

\section{References}

1. C. F. Bohren and D. R. Huffman, Absorption and Scattering of Light by Small Particles, John Wiley \& Sons, New York (1983).

2. E. M. Purcell and C. R. Pennypacker, "Scattering and absorption of light by nonspherical dielectric grains," Astrophys. J. 186, 705-714 (1973).

3. S. Oldenburg, R. Averitt, S. Westcott, and N. Halas, "Nanoengineering of optical resonances," Chem. Phys. Lett. 288(2-4), 243-247 (1998).

4. P. K. Jain, X. Huang, I. H. El-Sayed, and M. A. El-Sayed, "Noble metals on the nanoscale: Optical and photothermal properties and some applications in imaging, sensing, biology, and medicine," Acc. Chem: Res. 41, 1578-1586 (2008).

5. A. Alù and N. Engheta, "The quest for magnetic plasmons at optical frequencies," Opt. Express 17, 5723-5130 (2009).

6. A. Alù and N. Engheta, "Dynamical theory of artificial optical magnetism produced by rings of plasmonic nanoparticles," Phys. Rev. B 78, 085112 (2008).

7. N. A. Mirin and N. J. Halas, "Light-bending nanoparticles," Nano Lett. 9, 1255-1259 (2009).

8. K. Huang, M. Povinelli, and J. Joannopoulos, "Negative effective permeability in polaritonic photonic crystals," Appl. Phys. Lett. 85, 543-545 (2004).

9. C. Holloway, E. Kuester, J. Baker-Jarvis, and P. Kabos, "A double negative (dng) composite medium composed of magnetodielectric spherical particles embedded in a matrix," IEEE Trans. Antennas Propagat. 51(10), 2596-2603 (2003).

10. O. Vendik and M. Gashinova, "Artificial double negative (dng) media composed by two different dielectric sphere lattices embedded in a dielectric matrix," 34th European Microwave Conference, Amsterdam 2004 (Horizon House Publications Ltd., Norwood, MA), Vol. 3, pp. 1209-1212 (2005).

11. M. Wheeler, J. Aitchison, and M. Mojahedi, "Three-dimensional array of dielectric spheres with an isotropic negative permeability at infrared frequencies," Phys. Rev. B 72, 193103 (2005).

12. V. Yannopapas and A. Moroz, "Negative refractive index metamaterials from inherently non-magnetic materials for deep infrared to terahertz frequency ranges," J. Phys.: Condens. Matter 17, 3717-3734 (2005).

13. A. Ahmadi and H. Mosallaei, "Physical configuration and performance modeling of all-dielectric metamaterials," Phys. Rev. B 77, 045104 (2008).

14. M. Wheeler, J. Aitchison, J. Chen, G. Ozin, and M. Mojahedi, "Infrared magnetic response in a random silicon carbide micropowder," Phys. Rev. B 79, 073103 (2009).

15. L. Jylhä, I. Kolmakov, S. Maslovski, and S. Tretyakov, "Modeling of isotropic backwardwave materials composed of resonant spheres," J. Appl. Phys. 99, 043102 (2006).

16. L. Peng, L. Ran, H. Chen, H. Zhang, J. Kong, and T. Grzegorczyk, "Experimental observation of left-handed behavior in an array of standard dielectric resonators," Phys. Rev. Lett. 98, 157403 (2007).

17. J. Schuller, R. Zia, T. Taubner, and M. Brongersma, "Dielectric metamaterials based on electric and magnetic resonances of silicon carbide particles," Phys. Rev. Lett. 99, 107401 (2007).

18. K. Vynck, D. Felbacq, E. Centeno, A. Cuabuz, D. Cassagne, and B. Guizal, "All-dielectric rod-type metamaterials at optical frequencies," Phys. Rev. Lett. 102, 133901 (2009).

19. T. G. Mackay, "Lewin's homogenization formula revisited for nanocomposite materials," J. Nanophoton. 2, 029503 (2008). 
20. M. Kerker, D. Wang, and G. Giles, "Electromagnetic scattering by magnetic spheres," $J$. Opt. Soc. Am. 73, 765-767 (1983).

21. U. Leonhardt, "Optical conformal mapping," Science 312, 1777-1780 (2006).

22. J. Pendry, D. Schurig, and D. Smith, "Controlling electromagnetic fields," Science 312, 1780-1782 (2006).

23. A. Alù and N. Engheta, "How does zero forward-scattering in magnetodielectric nanoparticles comply with the optical theorem?," J. Nanophoton. 4, 041590 (2010).

24. A. Lakhtakia, V. K. Varadan, and V. V. Varadan, "Reflection and transmission of plane waves at the planar interface of a general uniaxial medium and free space," J. Mod. Optics 38, 649-657 (1991).

25. B. García-Cámara, F. Moreno, F. González, and O. J. F. Martin, "Light scattering by an array of electric and magnetic nanoparticles," Opt. Express 18, 10001-10015 (2010).

26. S. Albaladejo, R. Gómez-Medina, L. S. Froufe-Pérez, H. Marinchio, R. Carminati, J. F. Torrado, G. Armelles, A. García-Martín, and J. J. Sáenz, "Radiative corrections to the polarizability tensor of an electrically small anisotropic dielectric particle," Opt. Express 18, 3556-3567 (2010).

27. V. V. Temnov, G. Armelles, U. Woggon, D. Guzatov, A. Cebollada, A. García-Martín, J.-M. García-Martín, T. Thomay, A. Leitenstorfer, and R. Bratschitsch, "Active magnetoplasmonics in hybrid metalferromagnet structures," Nature Photon. 4, 107-111 (2010).

28. M. Nieto-Vesperinas, J. J. Sáenz, R. Gómez-Medina, and L. Chantada, "Optical forces on small magnetodielectric particles," Opt. Express 18, 11428-11443 (2010).

29. M. Nieto-Vesperinas, R. Gómez-Medina, and J. J. Sáenz, "Angle-suppressed scattering and optical forces on submicrometer dielectric particles," J. Opt. Soc. Am. A 28, 54-60 (2011).

30. A. E. Miroshnichenko, "Non-Rayleigh limit of the Lorenz-Mie solution and suppression of scattering by spheres of negative refractive index," Phys. Rev. A 80, 013808 (2009).

31. B. García-Cámara, F. Moreno, F. González, J. M. Saiz, and G. Videen, "Light scattering resonances in small particles with electric and magnetic properties," J. Opt. Soc. Am. A 25, 327-34 (2008).

32. A. Evlyukhin, C. Reinhardt, A. Seidel, B. Luk'yanchuk, and B. Chichkov, "Optical response features of Si-nanoparticle arrays," Phys. Rev. B 82(4), 045404 (2010).

33. A. García-Etxarri, R. Gómez-Medina, L. S. Froufe-Pérez, C. López, L. Chantada, F. Scheffold, J. Aizpurua, M. Nieto-Vesperinas, and J. J. Sáenz, "Strong magnetic response of submicrometer silicon particles in the infrared," Opt. Express 19, 4815-4826 (2011).

34. R. Gómez-Medina, M. Nieto-Vesperinas, and J. J. Sáenz, "Nonconservative electric and magnetic optical forces on submicrometer dielectric particles," Phys. Rev. A 83, 033825 (2011).

35. E. D. Palik, Handbook of Optical Constants of Solids, Academic Press, Orlando, Florida (1985).

36. P. Chaumet and A. Rahmani, "Electromagnetic force and torque on magnetic and negative-index scatterers," Opt. Express 17(4), 2224-2234 (2009).

37. G. Volpe, R. Quidant, G. Badenes, and D. Petrov, "Surface plasmon radiation forces," Phys. Rev. Lett. 96, 238101 (2006).

38. S. Albaladejo, M. I. Marqués, F. Scheffold, and J. J. Sáenz, "Giant enhanced diffusion of gold nanoparticles in optical vortex fields," Nano Lett. 9, 3527-3531 (2009).

39. S. Albaladejo, M. I. Marqués, M. Laroche, and J. J. Sáenz, "Scattering forces from the curl of the spin angular momentum of a light field," Phys. Rev. Lett. 102, 113602 (2009).

40. R. Gómez-Medina, P. S. José, A. García-Martín, M. Lester, M. Nieto-Vesperinas, and J. J. Sáenz, "Resonant radiation pressure on neutral particles in a waveguide," Phys. Rev. Lett. 86, 4275-4277 (2001).

Biographies and photographs of the authors not available. 